\title{
NUMERICAL STUDY OF A \\ GRAVITATING SYSTEM OF COLLIDING PARTICLES; FORMATION AND DYNAMICS OF DISCS
}

\author{
A. BRAHIC \\ Observatoire de Paris, Meudon, Université Paris VII, Paris, France
}

\begin{abstract}
The study of gravitating systems of colliding particles has many potential astrophysical applications, for instance the dynamics of Saturn's ring, the formation of the solar system, the flattening of protogalaxies and the evolution of galactic nuclei. We consider numerically a three-dimensional system of particles moving in the gravitational field of a central mass point and interacting through inelastic collisions. After a very fast flattening, the system forms a disc of finite thickness: this disc spreads slowly, and collisions still occur. A central condensation is formed and there is an outward flux of angular momentum. The energy which is continually lost in the inelastic collisions is obtained at the expense of the bodies which fall inwards.
\end{abstract}

There can be little doubt that collisions between 'macroscopic bodies' are of frequent occurrence in the Universe. All kinds of quite different objects undergo such collisions - these may range from interstellar clouds to small solid bodies in the solar system; it is therefore important to understand the past and present contribution of collisions to the overall evolution of the system in which they take place.

It has been known for a long time that inelastic collisions tend to flatten any system (Poincaré, 1911).

The configuration of a flat disc around a central body or bulge is found in many different contexts, for example spiral galaxies, the solar system, Saturn's ring, planetary satellites, etc. It may well be that the disc is often produced by the effect of contraction on a rotating mass of gas: nevertheless, it is interesting to see just what could be the effect of other mechanisms - in particular of inelastic collisions.

Therefore, in view of the many possible astrophysical applications, it would seem important to investigate gravitating systems of colliding particles. A full dynamical study has never been completed. A numerical study seems more suitable for this than an analytical formulation based on the Boltzmann equation, because such a formulation involves too many approximations of uncertain effect, even though a number of interesting conclusions can be derived from it for some specific cases.

Calculations of this sort have so far only been carried out by Ulam (1968), who was interested in the nuclei of galaxies, and by Trulsen $(1972 \mathrm{a}, \mathrm{b})$ who studied the dynamics of 'jet streams'. Corresponding numerical experiments have stimulated significant progress in molecular dynamics (Alder and Wainwright, 1959, 1960; Rahman, 1964; Verlet, 1967, 1968).

We hope thereby to throw some light on a number of still unanswered questions:

What is the evolution of such a system in the limit of very large times?

What is the time scale of evolution under the effect of collisions?

Does the system reach an equilibrium state? or not?

What is the energy balance? 
Are inelastic collisions sufficient to explain the formation and dynamics of some discs in the Universe? or do they constitute merely a secondary process?

With the help of Michel Hénon, I am studying systematically these kinds of systems by numerical simulation. This work is not only a way of finding the winning strategy in a game of three-dimensional billiards, but finds an immediate application to the as yet poorly understood dynamics of Saturn's ring (Jeffreys, 1947; Cook et al., 1972; Brahic, 1974b, 1975). Note that this is a case where on the one hand, the input of the theory is relatively free of uncertainties, and on the other hand, detailed observations will be soon available, so that observation and theory can be compared fruitfully. Of course, many other astrophysical potential applications suggest themselves, for example:

The dynamics of colliding cloudlets, such as have been invoked to explain the formation of the solar system disc, and the disc of planetary satellites (McCrea, 1960; Woolfson, 1964; Urey, 1966).

Another example could be the dynamics of protostars in a cluster: it has been suggested that collisions of protostars in a gas cloud which has just fragmented could affect their subsequent evolution (Arny and Weissman, 1973).

One might also study collisions in galactic nuclei (Spitzer and Saslaw, 1966; Spitzer and Stone, 1967; Sanders, 1970).

To explain the generation of X-ray sources in close binary systems, Prendergast and Burbidge (1968), Schwartzman (1971), Pringle and Rees (1972), and others introduce a model in which matter form a differentially rotating disc around a compact object - either a neutron star or a black hole. A somewhat similar situation, but on a much larger scale, is discussed by Lynden-Bell (1969) and by Lynden-Bell and Rees (1971), with application to quasars and active galactic nuclei in which gas clouds collide to form a nuclear disc.

Finally, we note also that Brosche (1970) assumed that a protogalaxy consists of several randomly moving clouds. The galaxy loses energy through collisions and therefore shrinks. Brosche made an approximate qualitative and analytical model of this process and found that the Hubble sequence could be interpreted as angular momentum sequence at constant mass. He also noted that this kind of model could be refined by an $N$-body calculation.

I am currently studying a sequence of numerical models. The system under study is evidently an $N$-body system, but, for the time being, my first models in no way utilise $N$-body techniques. I intend to include some kind of $N$-body integration using Aarseth's method (1973), for example - at a future date.

The pure dynamics of a gravitating system of colliding particles is by no means obvious. In order to understand first the basic mechanics of the process, I consider the simplest model. Attraction between particles has been neglected; and so particles orbits are keplerian around a central mass point. Positions and velocities at any given time are obtained from Kepler's equation. In a collision, the grazing component of velocity is conserved and the perpendicular component is multiplied by a coefficient $k$ which lies between 0 and $-1 ;-1$ corresponding to the elastic case. The 


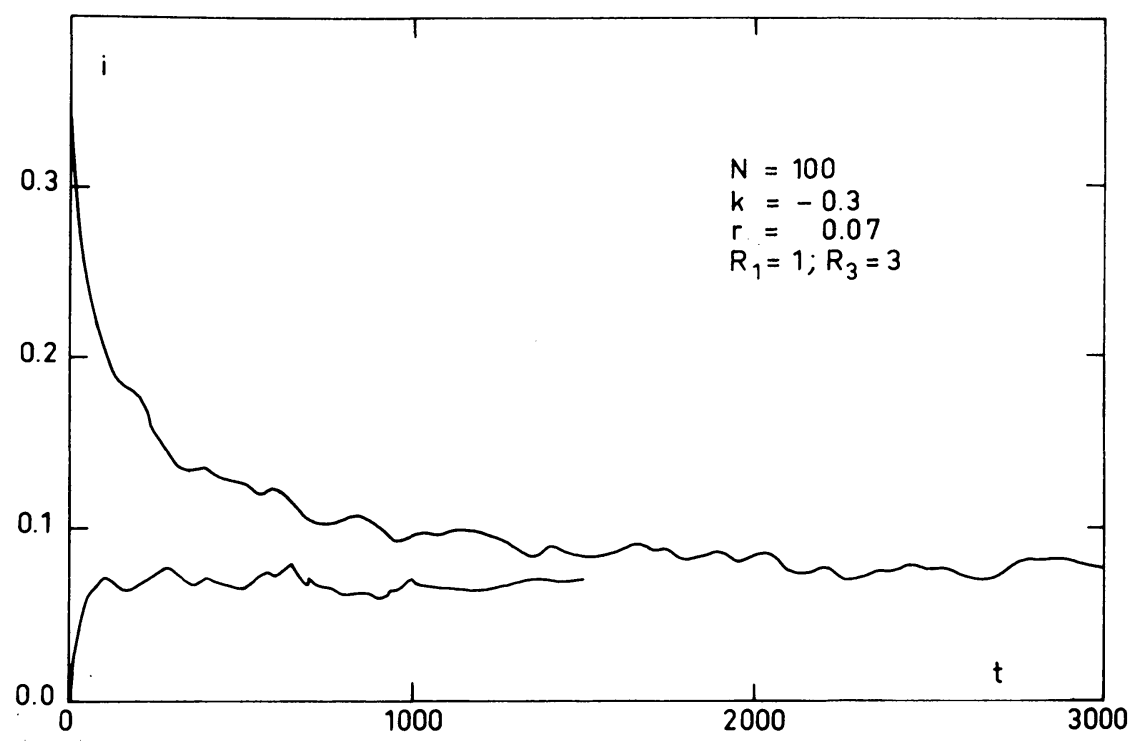

Fig. 1. Variations as a function of time of the mean inclination. The number of particles is equal to $N=100$ and their radius to $r=0.07$. Initial trajectories are all ellipses, which lie. between two spheres of radius $R_{1}=1$ and $R_{2}=3$ respectively and centered on the central mass point, and with inclinations lying between 0 and $0.5 \mathrm{rad}$ (for the upper curve) and between 0 and $10^{-3} \mathrm{rad}$. (for the lower curve). In the case of the upper curve, after 5257 collisions $(t=3000), 1$ body out of 100 has escaped and 23 out of 100 have fallen on the central body.

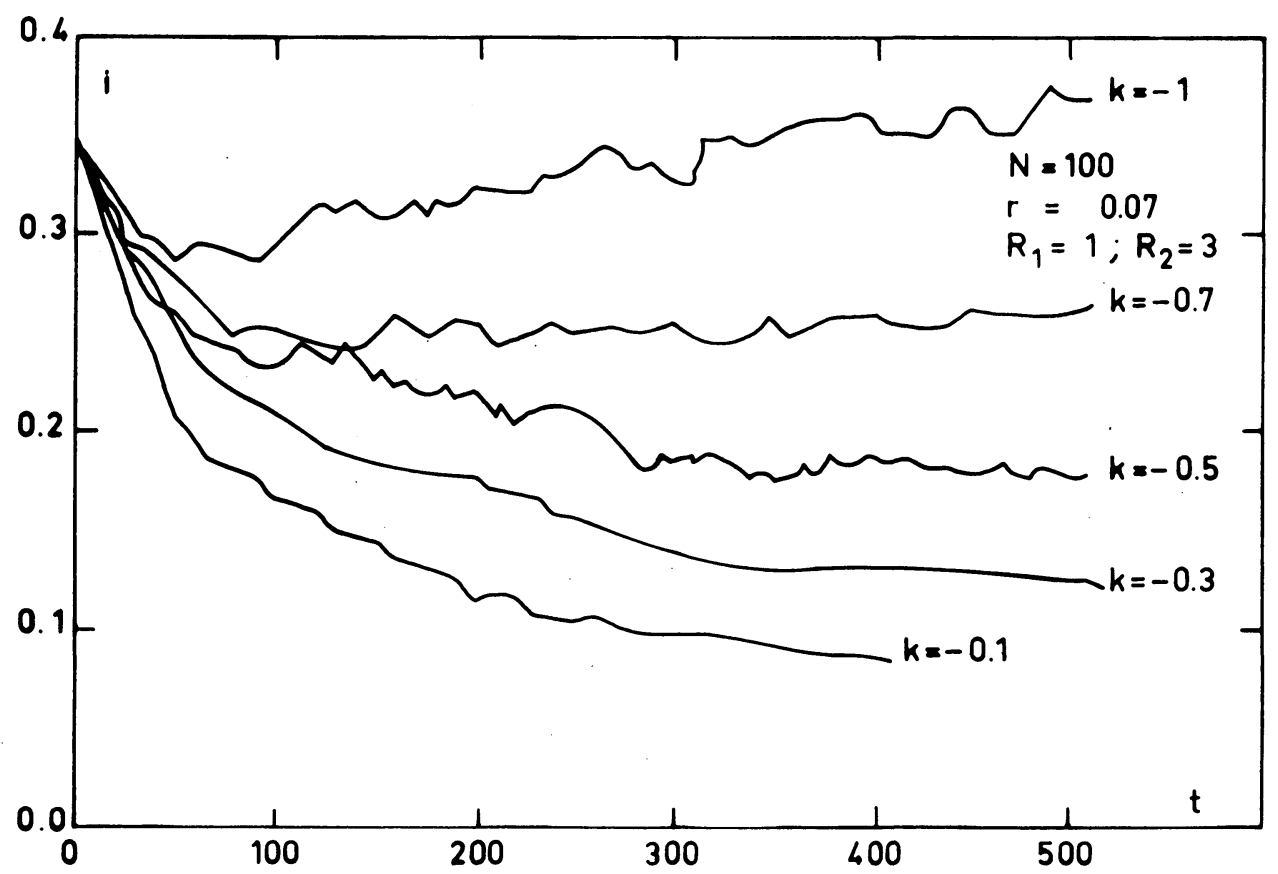

Fig. 2. Variations as a function of time of the mean inclination for different values of the rebound coefficient $k$. The initial inclinations of the orbits are all distributed between 0 and $0.5 \mathrm{rad}$. 


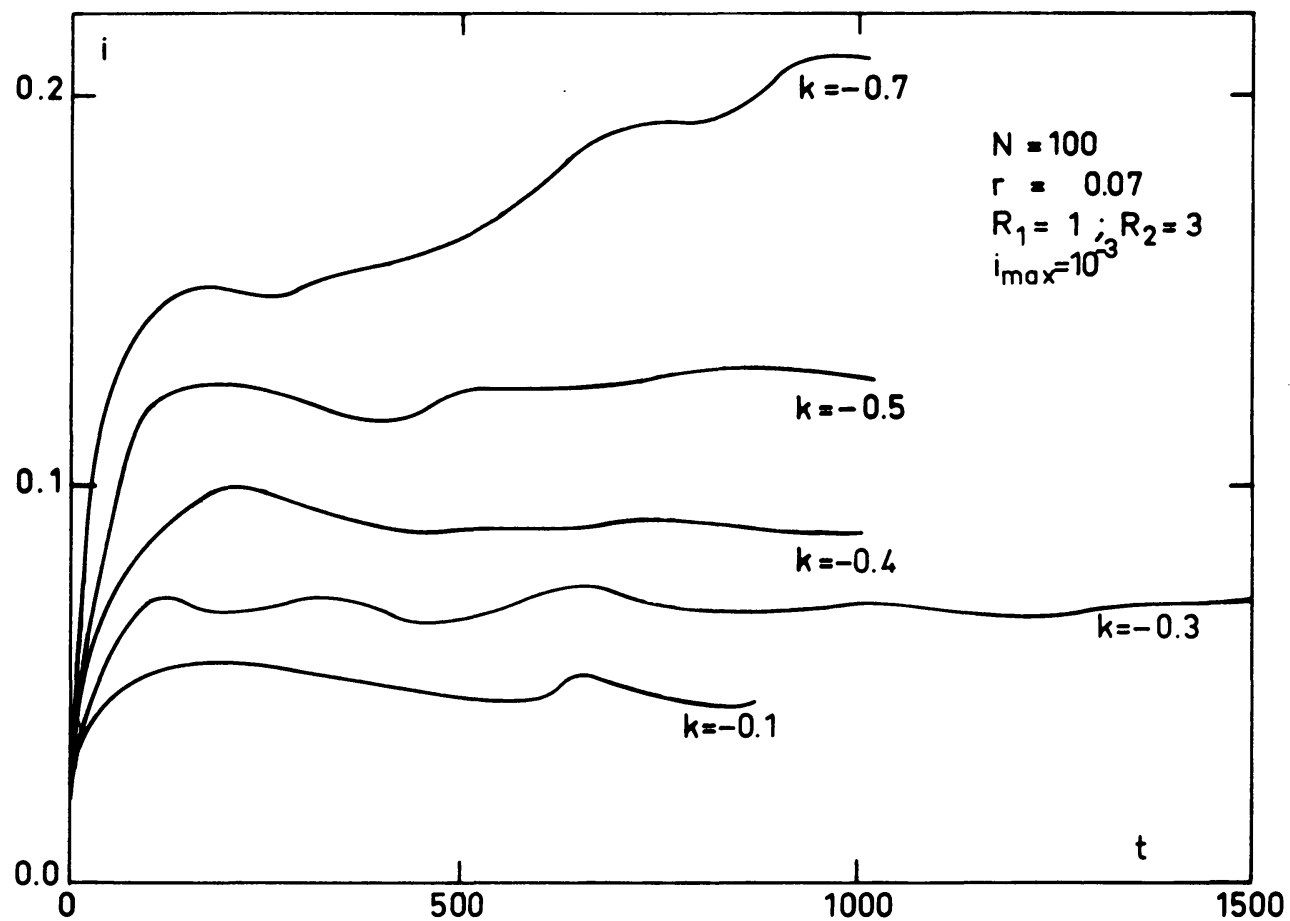

Fig. 3. Variations as a function of time of the mean inclination for different values of the rebound coefficient $k$. The initial inclinations of the orbits are all distributed between 0 and $10^{-3} \mathrm{rad}$.

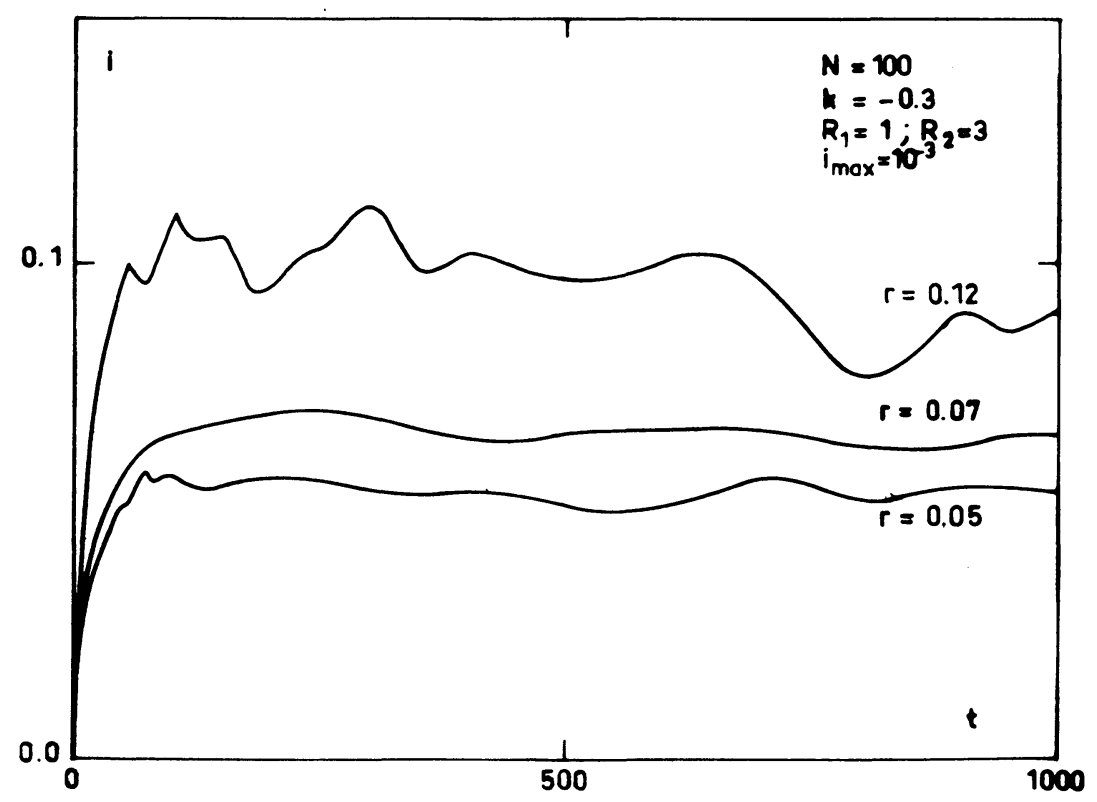

Fig. 4. Variations as a function of time of the mean inclination for different values of the size $r$ of the particles. The initial inclinations of the orbits are all distributed between 0 and $10^{-3} \mathrm{rad}$. 
initial conditions were set up by selecting at random the six elements of the keplerian orbit in such a way that trajectories were all ellipses lying between two spheres centered on the central mass point and with inclinations lying between 0 and some maximal value. We have assumed that particles on hyperbolic trajectories escape at once and, for technical reasons, that particles near to the centre are captured by the centre of mass. The kinetic rotational energy of the bodies has been neglected. The principal difficulty of the rather intricate calculations is to know whether two particles will in fact collide or not. By appropriate scaling, a few hundred particles suffice to simulate more realistic systems. Indeed, the time scale of evolution is inversely proportional to the number of particles and to their geometrical cross-section.

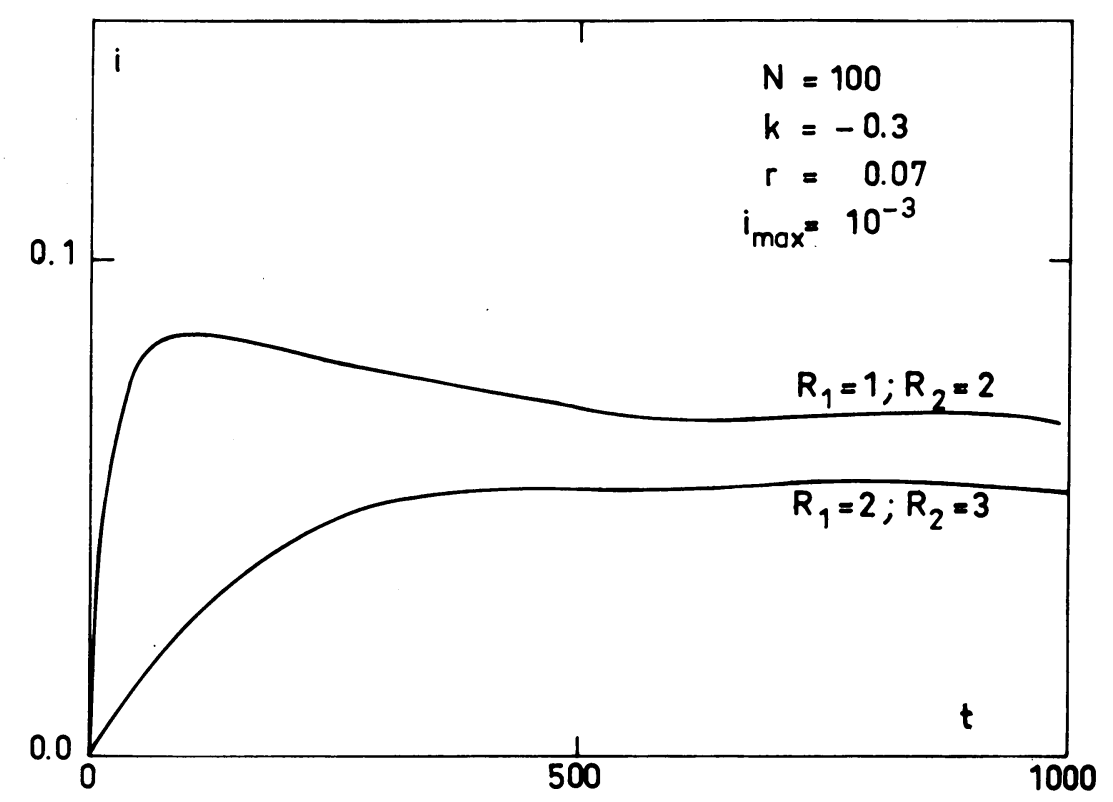

Fig. 5. Variations as a function of time of the mean inclination for different values of the scale size of the disc. The initial inclinations of the orbits are all distributed between 0 and $10^{-3} \mathrm{rad}$ and initial trajectories are all ellipses, which lie between two spheres of radius $R_{1}$ and $R_{2}$ respectively and centered on the central mass point.

The preliminary results were presented at the IAU symposia last year (Brahic, 1974a, b) and a number of more up to date results were presented this year (Brahic, 1975) within the context of the dynamics of Saturn's ring. I shall give only some examples of the results, repeating a few already presented, and summarise the most important points.

Figure 1 shows the mean inclination as a function of time in a typical case. As one would expect, the system is considerably flattened quickly - in less than about ten collisions per particle - but, contrary to what is often stated, collisions do not reduce the thickness of the disc to one layer of particles; after a fast flattening, the system reaches a quasi-equilibrium state (see the upper curve of Figure 1) in which the thick- 
ness is finite. This is presumably a consequence of the keplerian motion, which introduces differential rotation. Even if particles are in circular orbits, there are still collisions. Part of this residual velocity is transferred in vertical motion. It is exactly like viscosity which produces heat.

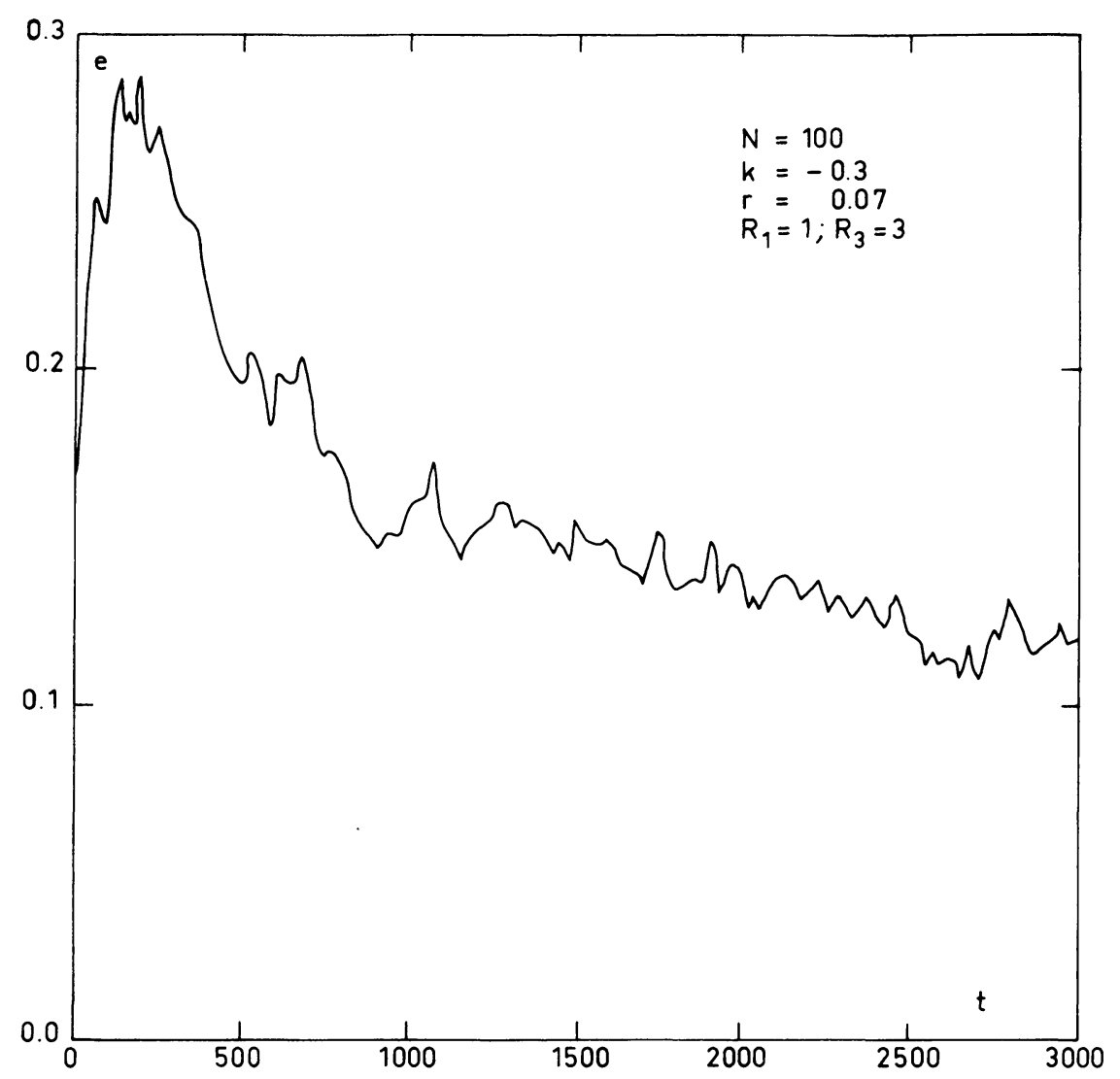

Fig. 6. Variations as a function of time of the mean eccentricity. The initial values of $N, r, R_{1}, R_{2}$, etc. are the same as for the upper curve of Figure 1.

If we start with a system almost completely flat (see the lower curve of Figure 1), the system is growing and reaches quickly the same limit; indeed, in a system where particles have finite collision cross-section, collisions necessarily introduce movements out of any plane as well as radial ones. We can start with a very small value of the mean inclination $i$ and study the limit as a function of the parameters of the problem.

The results are very sensitive to the rebound coefficient $k$ (see Figures 2 and 3). If $k$ lies between -0.5 and -1 , there is no important flattening of the system.

Figures 4 and 5 show how the ratio $r / R$ - where $r$ is the particle size, and $R$ is a scale size of the disc - affects the results. 
Figure 6 shows the mean eccentricity as a function of time for a typical case. The initial rise is due to the fact that thermal equilibrium is established between radial and vertical velocities. After this the orbits tends to become increasingly circular but the mean eccentricity does not reach 0 . This behaviour is similar to that of the mean inclination.

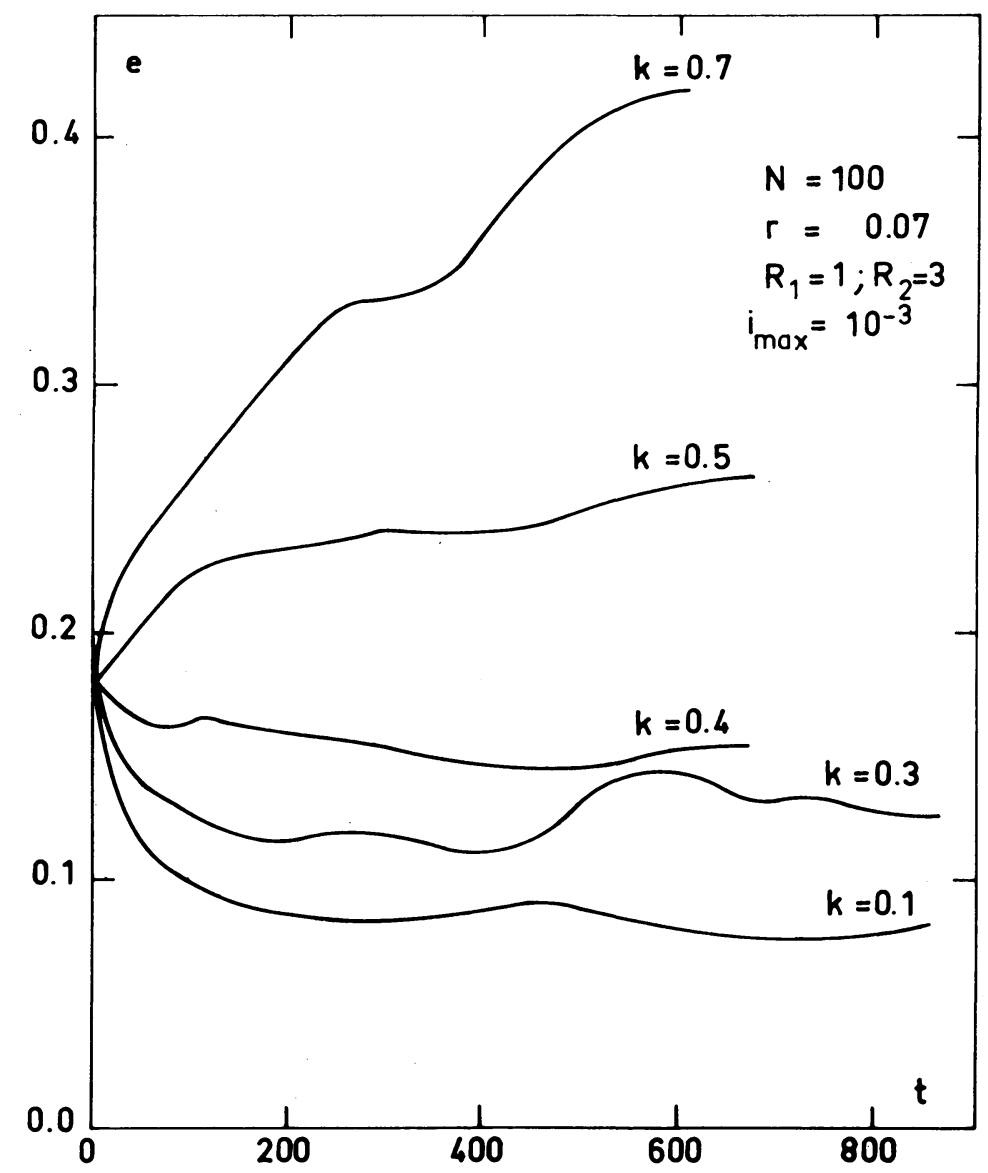

Fig. 7. Variations as a function of time of the mean eccentricity for different values of the rebound coefficient $k$.

Figures 7 and 8 show the role of the rebound coefficient $k$ and of the mean size of the particles respectively.

We have also started a study of other models; in particular if we consider particles of different masses and dimensions, the first results indicate that the very massive bodies were confined to a thinner disc than the light ones. Subsequent models will explore different kinds of collision regimes, fragmentation and coalescence and also the use of different potential field.

For the time being, the most interesting result is that an initial phase during which 


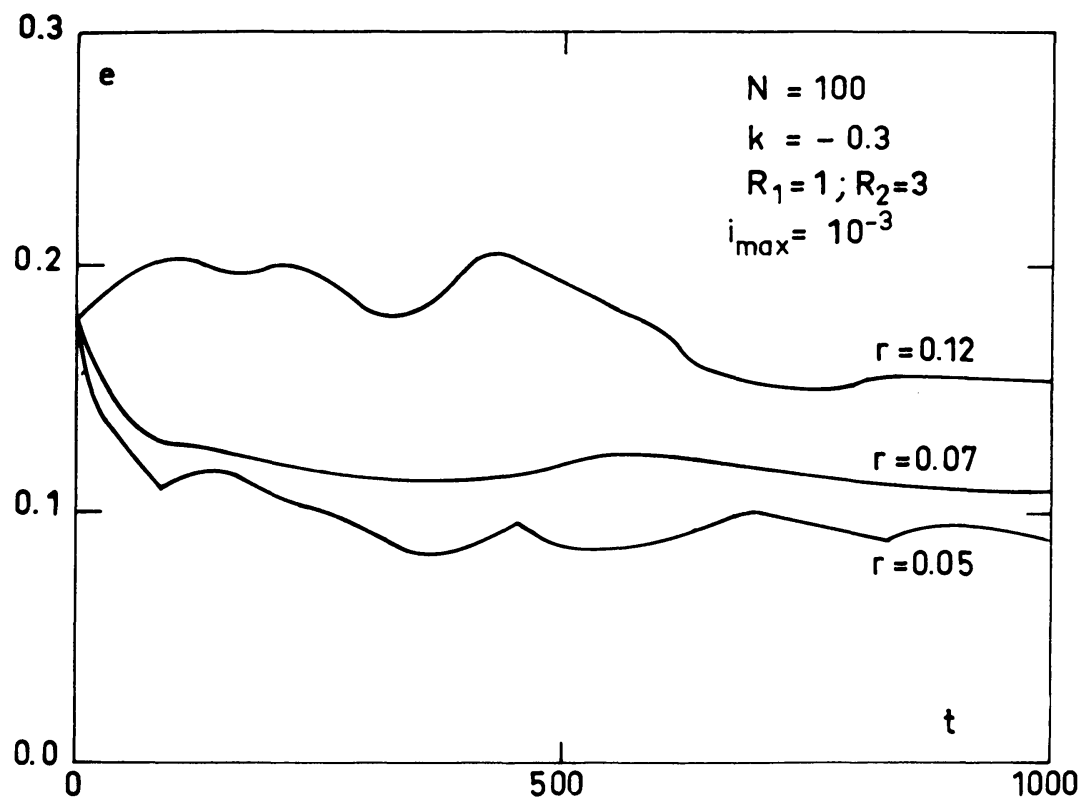

Fig. 8. Variations as a function of time of the mean eccentricity for different values of the size $r$ of the particles.

the system flattens rapidly is followed by a phase in which collisions still occur: the thickness of the disc thus formed is finite and the disc spreads very slowly: many particles move inwards forming a central condensation while some particles move outwards. There is an outward flux of angular momentum and the energy which is continually lost in the inelastic collisions is obtained at the expense of the bodies which fall inwards.

\section{References}

Aarseth, S. J.: 1973, Vistas in Astronomy 15, 13.

Alder, B. J. and Wainwright, T. E.: 1959, J. Chem. Phys. 31, 459.

Alder, B. J. and Wainwright, T. E.: 1960, J. Chem. Phys. 33, 1439.

Arny, T. and Weissmann, P.: 1973, Astron. J. 78, 309.

Brahic, A.: 1974a, in J. R. Shakeshaft (ed.), 'The Formation and Dynamics of Galaxies', IAU Symp. 58, 173.

Brahic, A.: 1974b, in Y. Kozai (ed.), 'The Stability of the Solar System and of Small Systems', IAU Symp. 62, 83.

Brahic, A.: 1975, in J. A. Burns (ed.), 'Planetary Satellites', IAU Colloq. No. 28; Icarus (in press).

Brosche, P.: 1970, Astron. Astrophys. 6, 240.

Cook, A. F., Franklin, F. A., and Palluconi, F. D.: 1973, Icarus 18, 317.

Jeffreys, H.: 1947, Monthly Notices Roy. Astron. Soc. 107, 263.

Lynden-Bell, D.: 1969, Nature 223, 690.

Lynden-Bell, D. and Rees, M. J.: 1971, Monthly Notices Roy. Astron. Soc. 152, 461.

McCrea, W. H.: 1960, Proc. Roy. Soc. A256, 245.

Poincaré, H.: 1911, Bull. Astron. 28, 251.

Prendergast, K. H. and Burbidge, G. R.: 1968, Astrophys. J. Letters 151, L83.

Pringle, J. E. and Rees, M. J.: 1972, Astron. Astrophys. 21, 1.

Rahman, A.: 1964, Phys. Rev. 136, 405.

Sanders, R. H.: 1970, Astrophys. J. 162, 791. 
Schwartzmann, V. F.: 1971, Soviet Astron. 15, 377.

Spitzer, L. and Saslaw, W. C.: 1966, Astrophys. J. 143, 400.

Spitzer, L. and Stone, M. E.: 1967, Astrophys. J. 147, 519.

Trulsen, J.: 1972a, Astrophys. Space Sci. 17, 241.

Trulsen, J.: 1972b, Astrophys. Space Sci. 18, 3.

Ulam, S. M.: 1968, Bull. Astron. 3ème serie 3, 265.

Urey, H. C.: 1966, Monthly Notices Roy. Astron. Soc. 131, 199.

Verlet, L.: 1967, Phys. Rev. 159, 98.

Verlet, L.: 1968, Phys. Rev. 165, 201.

Woolfson, M. M.: 1964, Proc. Roy. Soc. A282, 485.

\section{DISCUSSION}

Lecar: In the asteroid belt, there has been collisions and both the eccentricities and inclinations are large and approximately equal. In your model, are the final $Z$-dispersion velocities equal to the final $r$-dispersion velocities?

Brahic: Yes, the final $i$ and $e$ dispersions are of the same order of magnitude.

Lynden-Bell: What is the final value of the $e$-dispersion as a function of the parameters of the problem?

Brahic: It depends on the rebound coefficient $k$ and on $r / R$ where $r$ is the particle size and $R$ is a scale size of the disc. For $k=-0.3, r=0.07$ and $R=2, e \simeq 0.1$ which is the same order of magnitude as $i$. There is some kind of equipartition between the mean inclinations and the mean eccentricities.

Contopoulos: In some of your slides (e.g. when $k \leqslant-0.7$ ) there was a slow increase of inclination and eccentricity. How long does this increase continue?

Brahic: For $-1<k<-0.5$, I cannot really distinguish flattening from a quasi-equilibrium state: instead, what I see are rather large non-periodic fluctuations. I observe no important flattening for almost elastic collisions. 